Revue d'histoire de l'Amérique française

PGS REVUE D.HISTOIRE DE L'AMÉRIQUE FRANÇAISE

\title{
L'élection partielle du quartier-ouest de Montréal en 1832 : analyse politico-sociale
}

\section{France Galarneau}

Volume 32, numéro 4, mars 1979

URI : https://id.erudit.org/iderudit/303728ar

DOI : https://doi.org/10.7202/303728ar

Aller au sommaire du numéro

Éditeur(s)

Institut d'histoire de l'Amérique française

ISSN

0035-2357 (imprimé)

1492-1383 (numérique)

Découvrir la revue

Citer cet article

Galarneau, F. (1979). L'élection partielle du quartier-ouest de Montréal en 1832 : analyse politico-sociale. Revue d'histoire de l'Amérique française, 32(4), 565-584. https://doi.org/10.7202/303728ar d'utilisation que vous pouvez consulter en ligne.

https://apropos.erudit.org/fr/usagers/politique-dutilisation/ 


\title{
L'ÉLECTION PARTIELLE DU QUARTIER-OUEST DE MONTRÉAL EN 1832: ANALYSE POLITICO-SOCIALE
}

\author{
France GaLARNEAU \\ Québec
}

L'élection partielle du Quartier-Ouest de Montréal $^{1}$, tenue du 25 avril au 22 mai 1832, devint tristement célèbre à cause des incidents survenus le 21 mai $^{2}$. Trois Canadiens tombèrent sous les balles des soldats britanniques appelés sur les lieux par les magistrats. Le «massacre du 21 mai», comme La Minerve baptisa l'événement par la suite $^{3}$, se produisit le vingt-deuxième jour d'un suffrage où violences, intimidations réciproques et illégalités furent choses quotidiennes.

L'affrontement, à forte saveur ethnique, qui opposa Canadiens et Britanniques, découlait alors de tensions sociales, économiques et politiques, à l'image des autres conflits dans le Bas-Canada ${ }^{4}$. Il permit

1 Le Quartier-Ouest de Montréal, en 1832, est délimité au nord par la rue Craig; au sud, par le fleuve et la Petite Rivière; à l'ouest, par la rue McGill et la Place des Commissaires ainsi que la rue Ste-Radegonde; et à l'est, par la rue StJoseph (devenue depuis St-Sulpice) et la Place d'Armes. Pour plus de détails, voir le Rapport 1972-1973 du GRSM, p. 11.

2 Pour plus de détails sur les événements du 21 mai 1832, voir France Galarneau, L'élection partielle pour le Quartier-Ouest de Montréal en 1832: analyse politico-sociale, thèse de M.A. dactylographiée, Université de Montréal, 1978, chapitre III.

3 La Minerve fait mention à plusieurs reprises dans les mois suivants du « massacre du 21 mai».

4 L'une des manifestations de ces conflits est le sentiment de xénophobie, surtout anti-britannique, ressenti par la population francophone bas-canadienne. Ce phénomène prend toute sa dimension une fois relié à la lutte pour le pouvoir public. En effet, la bureaucratie qui dirige le pays est totalement étrangère au peuple canadien, qui se trouve exclu de presque tous les postes de prestige, du pouvoir et du patronage. Voir à ce sujet, Gilles Paquet et Jean-Pierre Wallot, Patronage et pouvoir dans le Bas-Canada (1794-1812): un essai d'économie historique (Montréal, Les 
aux principaux acteurs - les candidats, les électeurs et les groupes de pouvoir - de se manifester dans des rôles caractéristiques de la situation globale. L'élection se situa donc dans le prolongement logique des luttes opposant la Chambre d'Assemblée, majoritairement canadienne, aux Conseils, dont les membres, nommés par le gouverneur, étaient en grande majorité britanniques 5 .

Une étude des candidats, des voteurs et du déroulement de la votation s'impose afin d'éclaircir les faits.

\section{I. -L'élection: prélude à l'agitation du 21 mai 1832}

\section{A - Les candidats}

En 1832, le Quartier-Ouest de Montréal compte deux représentants à la Chambre d'Assemblée, chacun des électeurs intéressés ayant exprimé deux votes au moment de l'élection générale de 1830. Suite à cette dernière, John Fisher rejoignait en chambre LouisJoseph Papineau qui y siégeait depuis 1814. Mais, évoquant des raisons de santé, Fisher annonce sa démission le 26 mars $1832^{6}$. On émet alors à Québec, le 30 mars, un mandat pour une élection partielle fixée au 25 avril suivant ${ }^{7}$.

Aussitôt cette démission connue, les citoyens organisent de nombreuses assemblées afin de trouver des candidats. Ils en mettent officiellement trois en nomination: Stanley Bagg, Thomas Phillips et Daniel Tracey. Cependant, le 19 avril, soit six jours avant le début de l'élection, Thomas Phillips se désiste au profit de Bagg. Les lettres de présentation aux électeurs ${ }^{8}$ ont fait ressortir que les deux can-

Presses de l'Université du Québec, 1973). De plus, une rivalité économique contribue à isoler les Canadiens. $\grave{A}$ la ville, les anglophones sont proportionnellement plus nombreux qu'eux au sein des professions libérales (sauf pour le notariat), et des milieux d'affaires, avec pour résultat que les membres des professions libérales et le monde des affaires appuient majoritairement le candidat britannique. Voir Fernand Ouellet, Éléments d'histoire sociale du Bas-Canada (Montréal, Hurtubise HMH ltée, 1972) et id., Histoire économique et sociale du Québec, 1760-1850, tome II (Montréal, Fides, 1971).

5 Le principal sujet de plainte des Canadiens est que beaucoup de membres du Conseil législatif sont aussi membres du Conseil exécutif, créant ainsi une dépendance face à ce dernier, dépendance amenant le rejet de nombreux projets de loi présentés par l'Assemblée.

6 La Minerve, 26 mars 1832.

7 La Minerve, 12 avril 1832.

8 Ces lettres parurent dans La Minerve et la Montreal Gazette du 16 avril 1832. 
didats poursuivent les mêmes objectifs économiques et politiques, et que tous les deux travaillent à empêcher l'élection de Daniel Tracey au poste de représentant du Quartier-Ouest de Montréal. Une rencontre des deux comités ${ }^{9}$ amène finalement Phillips à se retirer de la course afin de renforcer l'opposition anti-canadienne. Quelques jours après l'élection, un «Anti-Aristocrate» réfléchissant sur ce désistement écrira:

Il n'avait pas été question de Bagg comme candidat dès le début de l'agitation pour l'élection. Le premier venu aurait plu au parti anti-canadien. M. Phillips vint s'offrir mais craignant que les Américains ne l'élisent pas, on décide de se servir de Bagg parce qu'il avait de l'argent à dépenser. ${ }^{10}$

Stanley Bagg, un Américain vivant au Canada depuis son enfance, bénéficie de l'appui de deux groupes d'électeurs, d'ailleurs très rapprochés: les Américains de Montréal, les Canadiens et Britanniques ${ }^{11}$ du parti bureaucrate. Tout l'élément anglophone de Montréal le soutient, excepté le groupe d'Irlandais favorable au docteur Tracey. De plus, certains Canadiens d'origine française s'ajoutent au nombre de ses partisans: certains membres de la bourgeoisie commerçante, tel Austin Cuvillier, ou encore des amis de l'ordre, comme P.-É. Leclère, le futur rédacteur de l'Ami du Peuple, de l'Ordre et des Lois ${ }^{12}$. Un marchand avance même, dans la Montreal Gazette, que les plus chauds partisans de Bagg comptent des «individus respectables et intelligents de naissance canadienne, craignant la disgrâce qui retomberait sur eux suite à l'élection d'un politicien tel que Tracey ${ }^{13}$. Ainsi, quelques Canadiens s'associent davantage aux propos de Bagg et du parti bureaucrate leur promettant des améliorations locales, notamment dans le secteur agricole, qu'à ceux de Tracey et du parti canadien visant à des réformes politiques qu'ils

9 Le comité de Bagg était formé de J. Fisher, H. Gates, A. Laframboise, P.É. Leclère, J.B. Castongué, R. Armour. Celui de Phillips réunissait J. Boston, J. Redpath, B. Hall, W. Bradbury, H. Driscoll, J. Jones et W. Ryan.

10 La Minerve, 31 mai 1832.

11 Tout au long de ce texte, les «Britanniques» désigneront les Anglais et les Écossais, cependant que les Irlandais demeureront un groupe à part.

12 Ce journal paraîtra en juillet 1832 et se caractérisera par une opposition très ouverte à La Minerve et par des propos des plus modérés concernant la politique et l'économie.

13 The Montreal Gazette, 26 avril 1832. 
considèrent dangereuses pour leurs intérêts ${ }^{14}$. Ainsi, une scission à l'intérieur du groupe canadien-français va à l'encontre des revendications du parti canadien à la Chambre d'Assemblée, division déterminée par des intérêts sociaux divergents plus forts, en ce cas, que la solidarité ethnique. En plus de représenter les commerçants anglais, Stanley Bagg est aussi l'allié des fonctionnaires britanniques et des rares Canadiens disposant d'une place dans l'administration. $L a$ Minerve affirme que ceux qui appuient Bagg se retrouvent parmi «les hommes qui [ont avoué] leur dessein de dominer les Canadiens ${ }^{15}$.

Stanley Bagg affronte Daniel Tracey, un médecin d'origine irlandaise, propriétaire du Vindicator, le seul journal anglophone de Montréal à prendre la défense des Canadiens. C'est son emprisonnement pour libelle diffamatoire envers le Conseil législatif, en janvier 1832, avec Ludger Duvernay, éditeur et rédacteur de La Minerve, qui a entraîné sa nomination comme candidat à l'élection partielle. Tracey représente les membres des professions libérales, en grand nombre à la Chambre, qui ont à lutter contre les intérêts des fonctionnaires britanniques, contre ceux aussi des marchands anglo-écossais et de leurs alliés canadiens. Le peuple cherchant à revendiquer ses droits à la terre et à l'emploi reconnaît aussi le respect que Tracey lui témoigne.

Il est possible de dire que Tracey dispose de l'appui d'une forte majorité des Canadiens français, notamment des classes populaires (paysans, ouvriers du bâtiment et de la construction navale, etc.) et des professions libérales (notaires, avocats, arpenteurs, etc.). Au contraire, les Britanniques de toutes classes soutiennent Bagg en bloc: la classe bureaucratique (gouverneur, conseillers législatifs et exécutifs), la bourgeoisie commerciale ainsi que celle des professions libérales (médecins, avocats, etc.), enfin, la classe populaire (colons anglais). Au cours de l'élection, les luttes entre le parti cana-

14 Trois grandes questions marquèrent la campagne de Tracey. La première fut celle de la liste civile, car sans le contrôle des deniers publics, les pouvoirs de la Chambre d'Assemblée paraissaient inutiles; la seconde, la composition du Conseil législatif qui aurait dû subir les «modifications qui en [auraient fait] un corps vraiment utile au Pays»; et la troisième, la présence de la Compagnie de Londres pour les Terres qui menaçait d'envoyer dans la province des colons anglais et écossais ne partageant pas nécessairement les visées de la majorité canadienne. À ce propos, voir La Minerve, 19 et 23 avril 1832.

is La Minerve, 23 avril 1832. 
dien et la coalition à la fois anti-canadienne et anti-Tracey s'expriment ouvertement. Les deux programmes présentés aux électeurs ont d'ailleurs laissé présager, de par leur contenu même, toute la violence, tout le caractère aigu de la contestation.

\section{B. - Le déroulement de l'élection}

Les seules exigences imposées aux électeurs sont les suivantes: avoir la qualité de sujet britannique, être âgé de vingt et un ans et répondre à certaines conditions matérielles. Pour les propriétaires, le cens électoral s'élève à $£ 5$, tandis que les locataires doivent payer un loyer annuel de $£ 10$ et être résidents du comté depuis un $a^{16}$. Quant aux électeurs féminins, seules les veuves, les célibataires et les femmes mariées en séparation de biens, qui satisfont aux obligations précédentes, peuvent voter. La durée de l'élection demeure indéterminée car le bureau de votation reste ouvert sept heures par jour, en moyenne, six jours par semaine, et ce, jusqu'à ce qu'une heure s'écoule sans qu'un seul vote soit exprimé. Tout se fait oralement et publiquement ${ }^{17}$.

Le lundi 25 avril 1832, Hyppolite St-Georges Dupré, l'officier rapporteur, ouvre officiellement la période de scrutin. Le tableau I, qui permet de suivre le déroulement du scrutin au jour le jour, montre Bagg avec une majorité de 23 voix en cette première journée. La présence de «bullies» joue sûrement en sa faveur ${ }^{18}$. Le mardi toutefois, un renversement se produit et Tracey jouit à son tour d'une avance de 22 votes. Le mercredi et le jeudi, 148 et 149 voteurs respectivement font leur choix, participant à l'affluence la plus forte de toute la contestation, signe d'un certain enthousiasme de la popula-

16 Ceci est valable pour les citadins. Quant aux habitants de la campagne, il leur suffisait d'être propriétaires d'une terre ayant une valeur annuelle de $£ 2$ en sus de toute rente à payer. Voir Denis Laforte et André Bernard, La législation électorale au Québec, 1760-1967 (Montréal, Les Éditions Sainte-Marie, 1969), 23.

17 Ibid., 53.

18 Les «bullies» sont «des fiers-à-bras engagés par un parti pour empêcher l'accès au poll aux partisans de l'adversaire». La manœuvre consiste pour un candidat à faire voter tôt le matin ses partisans, puis, quand il se dégage une majorité en sa faveur, ses «bullies» empêchent durant une heure les partisans de l'adversaire de s'approcher du poll. (Jean et Marcel Hamelin, Les mours électorales dans le Québec de 1791 à nos jours (Montréal, Les Éditions du Jour, 1962, p. 56) La présence de ces «bullies» nous est révélée par Jacques Viger, Statistique de l'élection de 1832, au Quartier-Ouest de la Cité de Montréal, ASQ, fonds Viger-Verreau, $\mathrm{n}^{\circ} 124$, liste $\mathrm{n}^{\circ} 12$, 19 (voir note 20 ). 
tion. Du 27 avril au 12 mai, les deux partis connaissent une situation délicate. Dès le 8 , la marge entre les deux adversaires se rétrécit, avec un écart qui n'est plus que de 32 voix. Tandis que les gains de Bagg demeurent assez stables, ceux de Tracey décroissent de façon importante. Puis, le 10 mai, Bagg obtient plus de votes que son opposant: 27 contre 23, mais l'avantage va encore à Tracey avec un total de 599 contre 581 . L'avance de 89 voix de ce dernier s'est réduite toutefois à 18 en huit jours. Elle est même ramenée à cinq le 11 mai, et à zéro, le 12 . Mais, le 14, les Canadiens rassemblent leurs énergies et avec 12 votes redonnent une majorité de deux voix à Tracey. Celui-ci réussit encore à se maintenir en avant le lendemain, 15 mai, même si peu de voteurs se présentent: six seulement pour lui, et sept pour Bagg. Le 16, Bagg gagne huit voix et Tracey sept ; seul fait notable, ce dernier perd sa majorité et les deux candidats se retrouvent sur un pied d'égalité avec chacun 658 votes. Le lendemain, par un retour en force, les Canadiens avec 14 votes permettent à Tracey de connaître une avance de cinq, Bagg ne recevant que neuf voix. Le 18 mai, trois votes s'ajoutent à ceux de Tracey et neuf à ceux de Bagg, qui obtient alors une majorité d'une voix. Le 19, les deux sont à nouveau sur un même pied avec 680 voix.

Le 21 mai revêt une importance particulière. Malgré les intimidations réciproques, dix électeurs votent pour Tracey et sept pour Bagg. Le début de la journée se déroule tranquillement, mais des altercations surviennent vers deux heures de l'après-midi entre quelques partisans, querelles tout à fait normales en période d'élection. A la clôture du bureau de votation, Tracey jouit d'une avance de trois, et le parti de Bagg ne semble plus pouvoir amener de voteurs. C'est à la fin de l'après-midi que des accrochages majeurs se produisent, à cause, semble-t-il, des chances très fortes de Tracey de remporter l'élection. Puis, le lendemain, un autre citoyen ajoute une voix au total de Tracey, ce qui confirme sa victoire par 691 à 687 , le vingt-troisième jour du scrutin. En tout, 1378 électeurs ont exprimé publiquement leur sympathie pour l'un ou l'autre des deux candidats ${ }^{19}$.

La lutte électorale, violente dès le début, atteint son paroxysme le 21 mai. Auparavant, des attaques menées de jour et de nuit

19 D'après Jacques Viger, Statistique de l'élection de 1832, au Quartier-Ouest de la Cité de Montréal, ASQ, fonds Viger-Verreau, $\mathrm{n}^{\circ} 124,31$. 
TABLEAU 1

DÉROULEMENT QUOTIDIEN DE L'ÉLECTION DE 1832

\begin{tabular}{|c|c|c|c|c|c|c|c|c|}
\hline \multirow[t]{2}{*}{ Dates } & \multirow{2}{*}{$\begin{array}{c}\text { Heures } \\
\text { d'ouverture } \\
\text { par jour }\end{array}$} & \multirow{2}{*}{$\begin{array}{c}\text { Nombre } \\
\text { de } \\
\text { voteurs } \\
\text { par jour }\end{array}$} & \multicolumn{2}{|c|}{ Votes } & \multicolumn{2}{|c|}{$\begin{array}{c}\text { Votes } \\
\text { cumulatifs }\end{array}$} & \multicolumn{2}{|c|}{ Majorité } \\
\hline & & & $\begin{array}{c}\text { pour } \\
\text { Tracey }\end{array}$ & $\begin{array}{l}\text { pour } \\
\text { Bagg }\end{array}$ & $\begin{array}{c}\text { pour } \\
\text { Tracey }\end{array}$ & $\begin{array}{l}\text { pour } \\
\text { Bagg }\end{array}$ & Tracey & Bagg \\
\hline $\begin{array}{l}25 \text { avril } \\
26 \\
27 \\
28 \\
30 \\
1 \text { er mai } \\
2 \\
3 \\
5 \\
7 \\
8 \\
9 \\
10 \\
11 \\
12 \\
14 \\
15 \\
16 \\
17 \\
18 \\
19 \\
21 \\
22\end{array}$ & $\begin{array}{l}41 / 2 \\
6^{1 / 2} \\
7 \\
7 \\
7 \\
7 \\
7 \\
7 \\
7 \\
7 \\
7 \\
7 \\
7 \\
7 \\
7 \\
7 \\
7 \\
7 \\
7 \\
7 \\
33 / 4 \\
9 \\
11 / 4\end{array}$ & $\begin{array}{r}123 \\
95 \\
148 \\
149 \\
129 \\
107 \\
91 \\
89 \\
61 \\
51 \\
41 \\
46 \\
50 \\
47 \\
39 \\
22 \\
13 \\
15 \\
23 \\
12 \\
9 \\
17 \\
1\end{array}$ & $\begin{array}{r}50 \\
70 \\
87 \\
88 \\
59 \\
66 \\
38 \\
33 \\
35 \\
21 \\
11 \\
18 \\
23 \\
17 \\
17 \\
12 \\
6 \\
7 \\
14 \\
3 \\
5 \\
10 \\
1\end{array}$ & $\begin{array}{l}73 \\
25 \\
61 \\
61 \\
70 \\
41 \\
53 \\
56 \\
26 \\
30 \\
30 \\
28 \\
27 \\
30 \\
22 \\
10 \\
7 \\
8 \\
9 \\
9 \\
4 \\
7 \\
-\end{array}$ & $\begin{array}{r}50 \\
120 \\
207 \\
295 \\
354 \\
420 \\
458 \\
491 \\
526 \\
547 \\
558 \\
576 \\
599 \\
616 \\
633 \\
645 \\
651 \\
658 \\
672 \\
675 \\
680 \\
690 \\
691\end{array}$ & $\begin{array}{r}73 \\
98 \\
159 \\
220 \\
290 \\
331 \\
384 \\
440 \\
466 \\
496 \\
526 \\
554 \\
581 \\
611 \\
633 \\
643 \\
650 \\
658 \\
667 \\
676 \\
680 \\
687 \\
687\end{array}$ & $\begin{array}{r}22 \\
48 \\
75 \\
64 \\
89 \\
74 \\
51 \\
60 \\
51 \\
32 \\
22 \\
18 \\
5\end{array}$ & 23 \\
\hline 23 jours & 151 hres & 1378 & 691 & 687 & 691 & 687 & $\begin{array}{l}\text { de } 1 \\
\text { à } 89\end{array}$ & $\begin{array}{l}\text { de } 1 \\
\text { à } 23\end{array}$ \\
\hline
\end{tabular}

Source: Jacques Viger, Statistique de l'élection, $A S Q$, fonds Viger-Verreau, $n^{\circ} 124$, 19-20.

ont donné lieu à des accusations mutuelles, et la force civile (officier rapporteur, magistrats) a subi des critiques acerbes de la part du parti canadien, comme nous le verrons plus loin. Seule une analyse des principales caractéristiques de l'élection et des électeurs per- 
met d'expliquer cette violence qui a conduit au «massacre du 21 mai».

\section{II. - Une analyse des électeurs, de l'élection et de la violence au poll}

\section{A - Une analyse des électeurs ${ }^{20}$}

L'origine ethnique des électeurs, ainsi que leur profession, expliquent la différence opposant les partisans de Bagg et ceux de Tracey. Ces deux éléments essentiels, combinés l'un à l'autre, permettent de mieux saisir le partage des votes lors de l'élection partielle de 1832; les tableaux 2 et 3 servent à l'illustrer ${ }^{21}$.

Ainsi, dans le tableau 2, la polarisation des voix, basée sur l'origine ethnique, saute aux yeux. Parmi les 1378 électeurs présents lors de la votation, 802 se disent canadiens ou irlandais, et 576 se déclarent d'extraction différente (britannique, américaine, allemande, italienne, etc.). Chez les Canadiens et les Irlandais, $81 \%$ appuient Tracey, et $19 \%$, Bagg. Ce dernier pourcentage est assez faible. Il peut s'agir souvent, dans leur cas, davantage d'une démonstration publique de fidélité à la Constitution britannique et au gouvernement que d'une attirance certaine pour ce candidat. Dans le contexte, approuver publiquement par un vote la conduite de Tracey pourrait passer pour une insoumission ou même une infidélité aux Conseils et à la Couronne. Par contre, la proportion des voteurs autres que canadiens et irlandais soutenant Bagg est très forte: elle s'élève à 93\%. Seulement $7 \%$ d'entre eux optent pour Tracey. Ainsi, les Canadiens et les Irlandais constituent toujours une majorité d'électeurs dans les diverses catégories professionnelles favorables au docteur

20 L'essentiel des renseignements pour l'étude des caractéristiques des électeurs du Quartier-Ouest de Montréal en 1832 provient des listes et statistiques laissées par Jacques Viger, contemporain de l'élection, qui les a dressées à partir du livre du poll. Cette Statistique de l'élection de 1832, au Quartier-Ouest de la Cité de Montréal est déposée dans le fonds Viger-Verreau, $n^{\circ} 124$, des Archives du Séminaire de Québec. À défaut du livre du poll pour l'élection partielle de 1832, qui est demeuré introuvable, les statistiques de Viger sont l'instrument le plus utile à consulter.

21 Les tableaux 2 et 3 sont construits à partir des listes les plus intéressantes préparées par Jacques Viger, à savoir celle de la «Distribution des personnes qui se sont présentées au Poll pour voter, d'après leur État, Profession ou Métier" (liste $\mathrm{n}^{\circ}$ 9), celle contenant les «Noms des Canadiens qui ont voté pour M. Bagg», ainsi que leur profession (liste $\mathrm{n}^{\circ} 6$ ), une autre indiquant les "Noms des Irlandais qui ont voté pour M. Bagg», avec leur profession (liste $\mathrm{n}^{\circ} 8$ ), et enfin, la liste des «Électeurs nés hors du Canada, ou d'extraction autre que canadienne qui ont voté pour M. Tracey», incluant leur profession. 
Tracey, tandis qu'ils sont toujours inférieurs en nombre au sein des professions gagnées à Bagg. Pour illustrer ce fait, prenons le groupe des hommes d'affaires où 223 voteurs choisissent Bagg et 148, Tracey. Dans ce cas, 197 personnes d'extraction autre que canadienne et irlandaise accordent leur préférence à l'Américain et seulement 26 Canadiens et Irlandais se joignent à eux. Toutefois, chez les artisans, 239 électeurs soutiennent Tracey et 200, Bagg. Les Canadiens et les Irlandais sont alors supérieurs numériquement par rapport aux voteurs d'origine ethnique différente, dont seulement 17 appuient Tracey. Ce tableau permet aussi de constater que tous les agriculteurs canadiens et irlandais suivent le docteur Tracey, et que les charretiers, de même que les journaliers, l'appuient aussi en grand nombre. Enfin, les fonctionnaires, les membres des professions libérales et les électeurs groupés dans la catégorie «divers» avantagent le candidat de l'administration au détriment du candidat patriote. Donc, la solidarité ethnique paraît plus forte chez les classes populaires, alors-que les intérêts sociaux sont proportionnellement plus solides parmi les classes plus instruites et probablement plus conscientes de leurs intérêts de classe.

Ce tableau montre aussi la relation ethnie/catégorie professionnelle ${ }^{22}$. Dans le domaine des affaires, la majorité des électeurs, soit $57 \%$, est d'origine ethnique autre que canadienne et irlandaise. Chez les artisans, l'inverse se produit car $62 \%$ d'entre eux sont nés au Canada ou en Irlande. Le phénomène se répète pour $73 \%$ des agriculteurs et $91 \%$ des charretiers. Ensuite, viennent les fonctionnaires avec un pourcentage égal de Canadiens/Irlandais et de voteurs d'autres origines. Enfin, les catégories professionnelles comprenant les journaliers, les membres des professions libérales et les divers comportent une forte majorité de Canadiens et d'Irlandais. Bref, c'est dans le seul secteur des affaires que la prédominance "étrangère», ou autre que canadienne et irlandaise, se manifeste.

Le tableau $3^{23}$, quant à lui, permet une analyse plus approfondie des liens existant entre l'origine ethnique des électeurs, leur profession et leur choix. Dans le groupe des affaires, la profession pour

22 Le calcul se fait ainsi: on additionne les deux nombres se rattachant à l'origine ethnique (ainsi, 26 et 133 Canadiens et Irlandais), à l'intérieur de chaque catégorie professionnelle. Ensuite, on fait le pourcentage par rapport au total. Dans le premier cas, $((26+133) \div 371) \times 100$.

23 La classification des diverses catégories professionnelles provient d'une communication sur les Poll-Books, donnée par Fernand Ouellet, à Chicoutimi en 1974. 
laquelle nous avons le plus grand nombre de participants à l'élection est celle des marchands. 105 d'entre eux votent pour Bagg, qui a axé sa campagne sur l'économie, et 26 appuient Tracey. Or, sur un total de 131, à peine 29 sont canadiens ou irlandais, et de ceuxlà, 11 seulement soutiennent Bagg. Par contre, les commerçants favorisent Tracey par 41 sur 49, alors que les aubergistes se partagent également entre les candidats et que les épiciers appuient Bagg en majorité. Les groupes plus nombreux parmi les artisans (menuisiers, cordonniers, charpentiers, forgerons, tailleurs) manifestent tous une préférence pour le candidat irlandais, hormis pour les charpentiers chez qui l'égalité des votes pourrait s'expliquer par l'assez grand nombre de voteurs d'origine ethnique autre que canadienne et irlandaise. Quant aux cultivateurs, peu nombreux, ils supportent majoritairement Tracey; il en est de même pour les charretiers. Chez les fonctionnaires, le groupe le plus nombreux, celui des instituteurs, à plus de $50 \%$, soutient le candidat de l'administration. Dans le groupe des journaliers, 36 Canadiens et Irlandais appuient Tracey sur une possibilité totale de 43 voteurs. Chez les membres des professions libérales, les avocats, qui disposent d'une plus grande chance de s'enrichir par comparaison aux cultivateurs, aux charretiers ou aux journaliers, se rangent du côté de Bagg, de l'argent et du commerce. D'ailleurs en 1831 à Montréal, 53\% d'entre eux sont anglophones ce qui pourrait expliquer en partie leur penchant pour le parti bureaucrate ${ }^{24}$.

Le tableau 4 établit les professions des électeurs de Tracey et de Bagg qui résident en-dehors de la cité de Montréal, mais qui répondent toutefois aux critères imposés par la loi. L'ensemble de ce groupe comprend surtout des bourgeois et des cultivateurs. Parmi ces derniers, 12 sur 15 votent pour Tracey, tandis que 12 bourgeois sur 18 appuient Bagg. Toutefois seulement-3,5\% des électeurs du parti bureaucrate et 5,3\% des partisans du parti patriote résident à l'extérieur de la cité de Montréal. Ces deux pourcentages sont faibles. Le tableau 5 précise les lieux de résidence de la majorité des électeurs.

Il est possible de connaître davantage les électeurs des deux candidats en ajoutant une autre dimension, celle de la "propriété». Ainsi, on peut départager les voteurs des deux partis en propriétaires et en locataires, en plus de préciser leurs lieux de résidence à l'intérieur de la cité de Montréal.

24 Fernand Ouellet, Éléments d'histoire sociale du Bas-Canada, 183. 
TABLEAU 2

\begin{tabular}{|c|c|c|c|c|c|c|c|c|c|}
\hline & Affaires & Artisans & $\begin{array}{l}\text { Agricul- } \\
\text { teurs }\end{array}$ & $\begin{array}{c}\text { Charre- } \\
\text { tiers }\end{array}$ & $\begin{array}{l}\text { Fonction- } \\
\text { naires }\end{array}$ & $\begin{array}{l}\text { Journa- } \\
\text { liers }\end{array}$ & $\begin{array}{c}\text { Membres des } \\
\text { professions } \\
\text { libérales }\end{array}$ & Divers & Total \\
\hline BAGG & & & & & & & & & \\
\hline $\begin{array}{l}\text { Canadiens \& } \\
\text { Irlandais } \\
\text { Autres } \\
\text { (incluant } \\
\text { Brit.) }\end{array}$ & $\begin{array}{r}26 \\
197\end{array}$ & $\begin{array}{r}50 \\
150\end{array}$ & 0 & 6 & $\begin{array}{r}4 \\
14\end{array}$ & 3 & $\begin{array}{l}11 \\
24\end{array}$ & $\begin{array}{r}52 \\
137\end{array}$ & $\begin{array}{l}152 \\
535\end{array}$ \\
\hline Total & 223 & 200 & 5 & 10 & 18 & 7 & 35 & 189 & 687 \\
\hline $\begin{array}{l}\text { TRACEY } \\
\text { Canadiens \& } \\
\text { Irlandais } \\
\text { Autres } \\
\text { (incluant } \\
\text { Brit.) }\end{array}$ & $\begin{array}{r}133 \\
15\end{array}$ & $\begin{array}{r}222 \\
17\end{array}$ & $\begin{array}{r}16 \\
1\end{array}$ & $\begin{array}{r}35 \\
0\end{array}$ & 0 & 34 & $\begin{array}{r}28 \\
0\end{array}$ & $\begin{array}{r}176 \\
6\end{array}$ & $\begin{array}{r}650 \\
41\end{array}$ \\
\hline Total & 148 & 239 & 17 & 35 & 6 & 36 & 28 & 182 & 691 \\
\hline $\begin{array}{l}\text { Grand } \\
\text { Total }\end{array}$ & 371 & 439 & 22 & 45 & 24 & 43 & 63 & 371 & 1378 \\
\hline
\end{tabular}


TABLEAU 3

\begin{tabular}{|c|c|c|c|c|c|c|c|c|}
\hline \multirow[t]{2}{*}{ Professions } & \multirow[t]{2}{*}{$\begin{array}{l}\text { Votes } \\
\text { Bagg }\end{array}$} & \multicolumn{3}{|c|}{$\begin{array}{l}\text { Origine } \\
\text { ethnique }\end{array}$} & \multirow[t]{2}{*}{$\begin{array}{l}\text { Votes } \\
\text { Tracey }\end{array}$} & \multicolumn{2}{|c|}{$\begin{array}{l}\text { Origine } \\
\text { ethnique }\end{array}$} & \multirow[t]{2}{*}{ Total } \\
\hline & & Can. & Irl. & Autres & & Can. \& Irl. & Autres & \\
\hline $\begin{array}{l}\text { Affaires } \\
\text { aubergiste }\end{array}$ & 23 & 3 & 2 & 18 & 23 & 21 & 2 & 46 \\
\hline boucher & 10 & 2 & 0 & 8 & 6 & 6 & 0 & $\begin{array}{l}40 \\
16\end{array}$ \\
\hline boulanger & 8 & 0 & 0 & 8 & 18 & 18 & 0 & 26 \\
\hline brasseur & 5 & 0 & 1 & 4 & 0 & 0 & 0 & 5 \\
\hline colporteur & 0 & 0 & 0 & 0 & 9 & 8 & 1 & 9 \\
\hline commerçant & 8 & 0 & 2 & 6 & 41 & 39 & 2 & 49 \\
\hline commis & 13 & 2 & 1 & 10 & 4 & 4 & 0 & 17 \\
\hline confiseur & 3 & 0 & 0 & 3 & 0 & 0 & 0 & 3 \\
\hline droguiste & 1 & 0 & 0 & 1 & 0 & 0 & 0 & 1 \\
\hline encanteur & 3 & 1 & 0 & 2 & 0 & 0 & 0 & 3 \\
\hline entrepreneur & 9 & 0 & 0 & 9 & 1 & 1 & 0 & 10 \\
\hline épicier & 23 & 1 & 0 & 22 & 15 & 14 & 1 & 38 \\
\hline hôtelier & 2 & 0 & 0 & 2 & 4 & 3 & 1 & 6 \\
\hline imprimeur & 3 & 0 & 0 & 3 & 0 & 0 & 0 & 3 \\
\hline libraire & 4 & 0 & 0 & 4 & 0 & 0 & 0 & 4 \\
\hline marchand \& & 105 & 5 & 6 & 94 & 26 & 18 & 8 & 131 \\
\hline modiste & 1 & 0 & 0 & 1 & 0 & 0 & 0 & 1 \\
\hline pâtissier & 1 & 0 & 0 & 1 & 0 & 0 & 0 & 1 \\
\hline tabaconiste & 1 & $\mathbf{0}$ & 0 & 1 & 1 & 1 & $\mathbf{0}$ & 2 \\
\hline TOTAL & 223 & 14 & 12 & 197 & 148 & 133 & 15 & 371 \\
\hline
\end{tabular}

Artisans armurier barbier bijoutier briqueteur brossier carossier chapelier charpentier c. de moulin c. de navire charron cloutier coiffeur cordonnier cuisinier doreur ébéniste f. chaises

f. moules

$\begin{array}{rl}1 & 0 \\ 1 & 1 \\ 1 & 0 \\ 7 & 0 \\ 4 & 0 \\ 3 & 0 \\ 3 & 0 \\ 17 & 3 \\ 4 & 0 \\ 0 & 0 \\ 2 & 0 \\ 2 & 0 \\ 3 & 0 \\ 17 & 2 \\ 1 & 1 \\ 1 & 0 \\ 16 & 1 \\ 1 & 0 \\ 2 & 0\end{array}$

$\begin{array}{rrr}0 & 1 & 3 \\ 0 & 0 & 0 \\ 1 & 0 & 0 \\ 0 & 7 & 0 \\ 0 & 4 & 0 \\ 0 & 3 & 1 \\ 0 & 3 & 2 \\ 2 & 12 & 17 \\ 0 & 4 & 0 \\ 0 & 0 & 1 \\ 0 & 2 & 8 \\ 0 & 2 & 0 \\ 0 & 3 & 0 \\ 11 & 4 & 29 \\ 0 & 0 & 3 \\ 1 & 0 & 0 \\ 2 & 13 & 3 \\ 0 & 1 & 0 \\ 0 & 2 & 0\end{array}$

$\begin{array}{rrr}3 & 0 & 4 \\ 0 & 0 & 1 \\ 0 & 0 & 1 \\ 0 & 0 & 7 \\ 0 & 0 & 4 \\ 1 & 0 & 4 \\ 1 & 1 & 5 \\ 17 & 0 & 34 \\ 0 & 0 & 4 \\ 1 & 0 & 1 \\ 8 & 0 & 10 \\ 0 & 0 & 2 \\ 0 & 0 & 3 \\ 25 & 4 & 46 \\ 1 & 2 & 4 \\ 0 & 0 & 1 \\ 3 & 0 & 19 \\ 0 & 0 & 1 \\ 0 & 0 & 2\end{array}$




\begin{tabular}{|c|c|c|c|c|c|c|c|c|}
\hline Professions & $\begin{array}{l}\text { Votes } \\
\text { Bagg }\end{array}$ & & $\begin{array}{l}\text { Origi } \\
\text { ethniq }\end{array}$ & & $\begin{array}{l}\text { Votes } \\
\text { Tracey }\end{array}$ & $\begin{array}{l}\text { Origin } \\
\text { ethniqu }\end{array}$ & & Total \\
\hline & & Can. & Irl. & Autres & & Can. \& Irl. & Autres & \\
\hline $\begin{array}{l}\text { f. pierres } \\
\text { de moulage }\end{array}$ & 0 & 0 & 0 & 0 & 1 & 1 & 0 & 1 \\
\hline $\begin{array}{l}\text { f. sas } \\
\text { f. toiles }\end{array}$ & 0 & 0 & 0 & 0 & 1 & 1 & 0 & 1 \\
\hline $\begin{array}{l}\text { cirées } \\
\text { f. toiles }\end{array}$ & 0 & 0 & 0 & 0 & 1 & 1 & 0 & 1 \\
\hline $\begin{array}{c}\text { peintes } \\
\text { facteur de }\end{array}$ & 1 & 1 & 0 & 0 & 0 & 0 & 0 & 1 \\
\hline pianos & 1 & 0 & 0 & 1 & 0 & 0 & 0 & 1 \\
\hline ferblantier & 3 & 0 & 1 & 2 & 5 & 5 & 0 & 8 \\
\hline fondeur & 4 & 0 & 0 & 4 & 1 & 1 & 0 & 5 \\
\hline forgeron & 15 & 0 & 2 & 13 & 18 & 18 & 0 & 33 \\
\hline horloger & 3 & 1 & 0 & 2 & 2 & 2 & 0 & 5 \\
\hline jardinier & 9 & 0 & 0 & 9 & 11 & 9 & 2 & 20 \\
\hline maçon & 7 & 4 & 0 & 3 & 15 & 15 & 0 & 22 \\
\hline marbrier & 1 & 0 & 0 & 1 & 0 & 0 & 0 & 1 \\
\hline menuisier & 4 & 2 & 0 & 2 & 45 & 44 & 1 & 49 \\
\hline $\begin{array}{l}\text { meunier } \\
\text { mouleur de }\end{array}$ & 1 & 0 & 0 & 1 & 0 & 0 & 0 & 1 \\
\hline bois & 1 & 1 & 0 & 0 & 0 & 0 & 0 & 1 \\
\hline palefrenier & 1 & 0 & 0 & 1 & 0 & 0 & 0 & 1 \\
\hline peignier & 1 & 0 & 0 & 1 & 0 & 0 & 0 & 1 \\
\hline peintureur & 5 & 0 & 0 & 5 & 11 & 9 & 2 & 16 \\
\hline pelletier & 0 & 0 & 0 & 0 & 2 & 1 & 1 & 2 \\
\hline perruquier & 1 & 1 & 0 & 0 & 0 & 0 & 0 & 1 \\
\hline piqueur & 1 & 0 & 0 & 1 & 0 & 0 & 0 & 1 \\
\hline plâtrier & 4 & 0 & 1 & 3 & 1 & 1 & 0 & 5 \\
\hline plombier & 3 & 0 & 0 & 3 & 0 & 0 & 0 & 3 \\
\hline $\begin{array}{l}\text { savonnier } \\
\text { scieur de }\end{array}$ & 4 & 0 & 1 & 3 & 5 & 5 & 0 & 9 \\
\hline long & 0 & 0 & 0 & 0 & 3 & 3 & 0 & 3 \\
\hline sellier & 5 & 0 & 1 & 4 & 7 & 7 & 0 & 12 \\
\hline tailleur & 14 & 0 & 2 & 12 & 17 & 16 & 1 & 31 \\
\hline t. de pierre & 1 & 0 & 1 & 0 & 0 & 0 & 0 & 1 \\
\hline maréchal & 0 & 0 & 0 & 0 & 1 & 0 & 0 & 1 \\
\hline tanneur & 4 & 0 & 1 & 3 & 14 & 14 & 0 & 18 \\
\hline teinturier & 1 & 0 & 0 & 1 & 0 & 0 & 0 & 1 \\
\hline tonnelier & 16 & 4 & 1 & 11 & 10 & 8 & 2 & 26 \\
\hline $\begin{array}{l}\text { tourneur } \\
\text { travailleur }\end{array}$ & 1 & 0 & 0 & 1 & 1 & 1 & 0 & 2 \\
\hline en cuivre & 2 & 0 & 0 & 2 & 0 & 0 & 0 & 2 \\
\hline TOTAL & 200 & 22 & 28 & 150 & 239 & 222 & 17 & 439 \\
\hline $\begin{array}{l}\text { Agriculteurs } \\
\text { cultivateur }\end{array}$ & 5 & 0 & 0 & 5 & 17 & 16 & 1 & 22 \\
\hline TOTAL & 5 & 0 & 0 & 5 & 17 & 16 & 1 & 22 \\
\hline
\end{tabular}




\begin{tabular}{llcccc} 
Professions & $\begin{array}{c}\text { Votes } \\
\text { Bagg }\end{array}$ & $\begin{array}{c}\text { Origine } \\
\text { ethnique }\end{array}$ & $\begin{array}{c}\text { Votes } \\
\text { Tracey }\end{array}$ & $\begin{array}{c}\text { Origine } \\
\text { ethnique }\end{array}$ & Total \\
\hline
\end{tabular}

Can. Irl. Autres Can. \& Irl. Autres

\begin{tabular}{lllllllll}
$\begin{array}{l}\text { Charretiers } \\
\text { charretier }\end{array}$ & 10 & 6 & 0 & 4 & 35 & 35 & 0 & 45 \\
\hline TOTAL & 10 & 6 & 0 & 4 & 35 & 35 & 0 & 45 \\
\hline $\begin{array}{l}\text { Fonctionnaires } \\
\text { capitaine } \\
\text { de port }\end{array}$ & 1 & 0 & 0 & 1 & 0 & 0 & 0 & 1 \\
$\begin{array}{l}\text { greffier } \\
\text { de paix }\end{array}$ & 1 & 1 & 0 & 0 & 0 & 0 & 0 & 1 \\
$\begin{array}{l}\text { huissier } \\
\text { instituteur }\end{array}$ & 1 & 0 & 1 & 0 & 0 & 0 & 0 & 1 \\
$\begin{array}{l}\text { inspecteur } \\
\text { bouuf salé }\end{array}$ & 2 & 0 & 1 & 7 & 5 & 5 & 0 & 13 \\
$\begin{array}{l}\text { i. potasse } \\
\text { i. thé } \\
\text { militaire }\end{array}$ & 2 & 1 & 0 & 2 & 0 & 0 & 0 & 2 \\
\hline TOTAL & 2 & 0 & 0 & 1 & 1 & 1 & 0 & 3 \\
\hline $\begin{array}{l}\text { Journaliers } \\
\text { journalier }\end{array}$ & 7 & 2 & 1 & 4 & 36 & 34 & 2 & 43 \\
\hline TOTAL & 7 & 2 & 1 & 4 & 36 & 34 & 2 & 43 \\
\hline
\end{tabular}

\begin{tabular}{|c|c|c|c|c|c|c|c|c|}
\hline $\begin{array}{l}\text { Professionnels } \\
\text { apothicaire }\end{array}$ & 1 & 0 & 0 & 1 & 1 & 1 & & \\
\hline $\begin{array}{l}\text { apotnicaire } \\
\text { architecte }\end{array}$ & 1 & 0 & 0 & 1 & 0 & 0 & 0 & 1 \\
\hline arpenteur & 1 & 0 & 0 & 1 & 1 & 1 & 0 & 2 \\
\hline avocat & 15 & 5 & 2 & 8 & 9 & 9 & 0 & 24 \\
\hline dentiste & 1 & 0 & 0 & 1 & 0 & 0 & 0 & 1 \\
\hline $\begin{array}{l}\text { en droit } \\
\text { étudiant }\end{array}$ & 0 & 0 & 0 & 0 & 1 & 1 & $\mathbf{0}$ & 1 \\
\hline $\begin{array}{l}\text { médecine } \\
\text { ingénieur }\end{array}$ & 0 & 0 & 0 & 0 & 1 & 1 & 0 & 1 \\
\hline civil & 2 & 0 & 0 & 2 & 1 & 1 & 0 & 3 \\
\hline médecin & 9 & 2 & 0 & 7 & 7 & 7 & 0 & 16 \\
\hline notaire & 4 & 2 & 0 & 2 & 7 & 7 & 0 & 11 \\
\hline protonotaire & 1 & 0 & 0 & 1 & 0 & 0 & 0 & 1 \\
\hline TOTAL & 35 & 9 & 2 & 24 & 28 & 28 & 0 & 63 \\
\hline $\begin{array}{l}\text { Divers } \\
\text { bedeau }\end{array}$ & 0 & 0 & 0 & 0 & 1 & 1 & 0 & 1 \\
\hline bibliothécaire & 1 & 0 & 1 & 0 & 0 & 0 & 0 & 1 \\
\hline bourgeois & 76 & 24 & 9 & 43 & 79 & 77 & 2 & 155 \\
\hline femme mariée & 12 & 0 & 0 & 12 & 6 & 6 & $\mathbf{0}$ & 18 \\
\hline & 26 & 6 & & 20 & 20 & 20 & 0 & 46 \\
\hline
\end{tabular}




\begin{tabular}{lrrrrrrrr} 
Professions & $\begin{array}{c}\text { Votes } \\
\text { Bagg }\end{array}$ & \multicolumn{2}{c}{$\begin{array}{c}\text { Origine } \\
\text { ethnique }\end{array}$} & $\begin{array}{c}\text { Votes } \\
\text { Tracey }\end{array}$ & \multicolumn{2}{c}{$\begin{array}{c}\text { Origine } \\
\text { ethnique }\end{array}$} & Total \\
& & Can. & Irl. & Autres & & Can. \& Irl. Autres & \\
maître & & & & & & & & \\
$\quad$ d'escrime & 0 & 0 & 0 & 0 & 1 & 0 & 1 & 1 \\
ministre & 4 & 0 & 0 & 4 & 0 & 0 & 0 & 4 \\
musicien & 1 & 0 & 0 & 1 & 0 & 0 & 0 & 1 \\
navigateur & 5 & 2 & 1 & 2 & 3 & 3 & 0 & 8 \\
pilote & 2 & 2 & 0 & 0 & 1 & 1 & 0 & 3 \\
veuve & 62 & 4 & 3 & 55 & 68 & 65 & 3 & 130 \\
voyageur & 0 & 0 & 0 & 0 & 3 & 3 & 0 & 3 \\
\hline TOTAL & 189 & 38 & 14 & 137 & 182 & 176 & 6 & 371 \\
\hline
\end{tabular}

Des 1378 électeurs qui se prévalent de leur droit de vote, soit en faveur de Bagg, soit au profit de Tracey, 730 s'identifient comme propriétaires et 648 , comme locataires. Qui, des deux candidats, attire le plus les propriétaires? Jacques Viger ${ }^{25}$ présente $60,3 \%$ des propriétaires votant pour le parti canadien, contre $39,7 \%$ pour le parti britannique, alors que $61,2 \%$ des locataires se rangent du côté de Bagg et 38,8\%, de celui de Tracey. Ces chiffres semblent, au premier abord, contredire les affirmations de la Montreal Gazette qui, durant la campagne électorale, ironisait sur la soi-disant grande pauvreté des électeurs de Tracey et sur le rejet de ce candidat par les gens respectables. Il serait peut-être possible de croire que c'est à cause de leur origine canadienne ou irlandaise que les propriétaires supportent majoritairement le parti canadien. Mais cela n'est pas prouvé. D'ailleurs, «la majorité des effectifs se concentr[ait] dans les propriétés à bas revenu, indice du désir des natifs du pays d'avoir une maison bien à eux, d'acheter un lot pour y vivre plutôt que pour y investir » ${ }^{26}$. Le fait que les Canadiens possèdent des propriétés n'est donc pas un indice de richesse en soi.

Parmi les groupes qui favorisent Tracey, on note les agriculteurs, les artisans, les charretiers et les journaliers. La majorité d'entre eux sont donc des propriétaires. Bagg s'appuie davantage sur les membres des professions libérales, les hommes d'affaires, artisans et fonctionnaires. À cause de lacunes dans les données laissées par

25 Jacques Viger, Statistique de l'élection de 1832, au Quartier-Ouest de la Cité de Montréal, 31.

26 Voir à ce sujet, P.-A. Linteau et J.-C. Robert, «Propriété foncière et société à Montréal: une hypothèse», $\operatorname{RHAF}(28,1$ (juin 1974)): 59. 
TABLEAU 4

PROFESSIONS DES ÉLECTEURS DE BAGG ET DE TRACEY, CHEZ CEUX QUI RÉSIDENT EN DEHORS DE LA CITÉ DE MONTRÉAL

\begin{tabular}{|c|c|c|c|}
\hline Profession & Vote pour Bagg & Vote pour Tracey & Total \\
\hline \multicolumn{4}{|l|}{ AFFAIRES: } \\
\hline $\begin{array}{l}\text { Boucher } \\
\text { Boulanger }\end{array}$ & - & 1 & 1 \\
\hline $\begin{array}{l}\text { Boulanger } \\
\text { Brasseur }\end{array}$ & $\overline{1}$ & 1 & $\begin{array}{l}1 \\
1\end{array}$ \\
\hline Marchand & 1 & 3 & 4 \\
\hline Total: & 2 & 5 & 7 \\
\hline \multicolumn{4}{|l|}{ ARTISANS: } \\
\hline Menuisier & - & 2 & 2 \\
\hline Tanneur & - & 3 & 3 \\
\hline Total: & - & 5 & 5 \\
\hline \multicolumn{4}{|l|}{ AGRICULTEURS : } \\
\hline Cultivateur & 3 & 12 & 15 \\
\hline \multicolumn{4}{|c|}{ PROFESSIONNELS: } \\
\hline Arpenteur & 1 & 1 & 2 \\
\hline Avocat & 1 & - & 1 \\
\hline Médecin & 1 & 1 & 2 \\
\hline Notaire & 2 & 1 & 3 \\
\hline Total: & 5 & 3 & 8 \\
\hline \multicolumn{4}{|l|}{ DIVERS : } \\
\hline Bourgeois & 12 & 6 & 18 \\
\hline Fille & - & 4 & 4 \\
\hline Veuve & 2 & 2 & 4 \\
\hline \multirow{2}{*}{ Total } & 14 & 12 & 26 \\
\hline & 24 & 37 & 61 \\
\hline
\end{tabular}

Source: Jacques Viger, Statistique de l'élection, 8.

Jacques Viger, nous devons nous contenter d'émettre des hypothèses sur les liens qui existent entre les deux faits mentionnés plus haut, en ce qui a trait aux locataires et aux propriétaires, d'une part, et à leurs catégories professionnelles, d'autre part. Nous pouvons croire que les marchands et les hommes d'affaires occupent des locaux situés en dehors de leurs lieux de résidence et qu'ils les louent. Les agriculteurs, charretiers et journaliers quant à eux ne manifestent aucun 
besoin de locaux situés dans les endroits passants de la ville: ils sont alors propriétaires de leurs maisons.

De quelles parties de la cité de Montréal ${ }^{27}$ viennent donc les électeurs de Bagg et de Tracey? Le tableau 5 indique une polarisation assez importante, compte tenu de la forte majorité dont un candidat jouit presque toujours par rapport à l'autre. Ainsi, Tracey est favori dans la partie ouest du faubourg St-Laurent, dans les faubourgs StAntoine et St-Joseph avec, respectivement, 53\%, 60,8\% et $74,1 \%$ des voix. Quant à Bagg, il domine dans les autres secteurs, soit: la partie ouest de la ville, le faubourg Ste-Anne et la Pointe-à-Callière, avec respectivement $66,3 \%, 76,8 \%$ et $60 \%$ des votes ${ }^{28}$. C'est dans le faubourg St-Laurent que la lutte entre les partisans de Bagg et ceux de Tracey est la plus chaude: $47 \%$ des électeurs sont favorables au premier; $53 \%$ appuient le dernier.

\section{TABLEAU 5}

\section{NOMBRE DE VOTEURS DU QUARTIER-OUEST DE MONTRÉAL PAR FAUBOURGS (SECTIONS)}

\begin{tabular}{l|r|r|r|r|c}
\hline $\begin{array}{l}\text { Faubourgs } \\
\text { (ou sections du }\end{array}$ & $\begin{array}{c}\text { Vote } \\
\text { pour } \\
\text { Quartier-Ouest) }\end{array}$ & $\begin{array}{c}\text { Pour- } \\
\text { centage }\end{array}$ & $\begin{array}{c}\text { Vote } \\
\text { pour } \\
\text { Tracey }\end{array}$ & $\begin{array}{c}\text { Pour- } \\
\text { centage }\end{array}$ & Total \\
\hline Partie ouest de la ville & 242 & $66,3 \%$ & 121 & $33,7 \%$ & 363 \\
Partie ouest du faubourg & 188 & $47,0 \%$ & 212 & $53,0 \%$ & 400 \\
$\quad$ St-Laurent & 72 & $40,2 \%$ & 112 & $60,8 \%$ & 184 \\
Faubourg St-Antoine & 103 & $25,8 \%$ & 209 & $74,1 \%$ & 282 \\
Faubourg St-Joseph & 9 & $60,0 \%$ & 31 & $23,1 \%$ & 134 \\
Faubourg Ste-Anne & 687 & & 691 & & 1378 \\
Pointe-à-Callière & & & & \\
\hline Total & & &
\end{tabular}

Source: Jacques Viger, Statistique de l'élection, 18.

27 Cité de Montréal et Ville de Montréal n'ont pas la même signification. La cité est «l'unité administrative délimitée et créée par la proclamation de 1792, et englobe la ville et les faugourgs». La ville est «l'espace circonscrit par le périmètre des anciennes fortifications; c'est la vieille ville du Régime français». Et, en 1827, il existe sept faubourgs: la Pointe-à-Callière, le faubourg Ste-Anne, celui de St-Joseph (ou des Récollets), le faubourg St-Antoine, le faubourg St-Laurent, le faubourg St-Louis et le faubourg Ste-Marie (ou Québec). Voir P.-A. Linteau et J.-C. Robert, «Propriété foncière et société à Montréal: une hypothèse», $\operatorname{RHAF}(28,1$ (juin 1974)): 51 ; idem, «Les divisions territoriales à Montréal au 19e siècle», Rapport 1972-1973 du GRSM.

28 Il demeure impossible pour le moment de connaître la proportion de Canadiens français et de Canadiens anglais, ainsi que des autres nationalités au sein de ces 


\section{B. - Une analyse de l'élection.}

L'élection partielle de 1832 a-t-elle été une élection libre? Oui, dans le sens où tous les électeurs répondant aux qualifications requises pouvaient s'avancer et exprimer leur préférence pour l'un des deux candidats. Mais, dans les faits, certains actes d'intimidation devinrent des entraves à la liberté des voteurs. Les électeurs qui ne votaient pas du même côté que leur patron risquaient la mise à pied et le chômage, ce qui n'empêcha pas des électeurs d'encourir ces risques. Ainsi, le Vindicator cite le cas de cinq employés au service de William Spiers, qui jouissait de contrats pour certains travaux publics municipaux, qui furent congédiés suite à leur vote en faveur de Tracey ${ }^{29}$.

Avec la présence de fiers-à-bras («bullies»), les intimidations physiques et les bagarres entre partisans des deux candidats étaient fréquentes. L'intimidation s'effectuait, semble-t-il, davantage aux dépens des partisans de Tracey que de ceux de Bagg. Ainsi, parmi les 60 personnes qui ont eu à se présenter au poll deux fois avant de voter $^{30}$, à cause la plupart du temps de menaces proférées contre elles, 39 ont voté pour Tracey et 21 pour Bagg. Au total, sur les 65 électeurs qui sont revenus sur le lieu de votation deux ou trois fois avant de pouvoir s'exprimer, 42 appuyaient Tracey et 23 , Bagg ${ }^{31}$. La présence au poll de connétables spéciaux et de magistrats chargés de maintenir la paix au profit de Bagg équivalait, selon le parti canadien, à une entrave à la liberté des électeurs. L'assermentation de ces connétables spéciaux se révéla un facteur primordial dans la montée de la violence au poll, par l'état d'exaspération qu'elle souleva parmi les partisans de Tracey. Une liste des connétables spéciaux assermentés pour l'élection, et commentée par Jacques Viger, mentionne que sur les 335 connétables spéciaux assermentés, 40 favorisaient Tracey, 213 , Bagg et que 82 étaient neutres ${ }^{32}$.

faubourgs. Le recensement de 1831 ne donne pas l'origine ethnique des habitants, et un recoupement à partir de la religion ne permet pas de la préciser davantage puisque tous les membres de la famille apparaissent et non pas seulement les chefs, les véritables électeurs.

29 The Vindicator, $1^{\text {er }}$ mai 1832. Il s'agit de John Tracey, Owen Murray, Samuel Finneron, James Mullaly et Edward Meaher.

30 Jacques Viger, Statistique de l'élection de 1832, au Quartier-Ouest de la Cité de Montréal, liste $\mathrm{n}^{\circ}$ 2, 3.

31 Ibid.

32 Ibid., liste $\mathrm{n}^{\circ} 15,23$. 
Les Canadiens, amis de Tracey, portaient aussi des accusations de partialité contre l'officier rapporteur. Sa conduite faible visait à protéger les magistrats et les connétables spéciaux ${ }^{33}$. Ils l'accusaient de se laisser manipuler par les amis de Bagg et de souffrir certaines illégalités; ainsi, il y aurait eu plus de votes rendus que le quartier était supposé en contenir ${ }^{34}$.

\section{Conclusion}

Le parti national l'a emporté sur la faction anticanadienne. Malgré la conduite faible et partiale de l'officier-rapporteur, malgré la violence, les menaces, la corruption, le parjure contre lesquels M. Tracey a eu à lutter, malgré la force militaire envoyée contre lui, malgré le massacre de trois Canadiens, malgré les canons braqués en face du lieu du poll, le tout à la demande des partisans de l'autre candidat, M. Tracey a été élu le vingt-troisième jour du poll. ${ }^{35}$

Des deux candidats qui s'opposèrent au cours de l'élection partielle d'avril et de mai 1832, le candidat du parti patriote, Daniel Tracey, fut appelé à siéger à la Chambre d'Assemblée du Bas-Canada. Il s'agissait à la fois d'un succès personnel et d'un succès politique puisqu'il venait de sortir de prison, où le Conseil législatif l'avait envoyé. Cette victoire rendait compte de l'appui que le peuple canadien lui assurait. Malheureusement, ce représentant choisi au prix de sanglants incidents mourut durant l'été de la même année. Emporté par le choléra, il n'eut l'occasion de siéger que durant quelques mois.

Le résultat de cette élection ne signifie pas seulement la victoire du parti canadien sur le parti britannique, et, d'une certaine façon sur Londres. Mais, plutôt, les Canadiens par leurs votes, marquèrent leur opposition à l'immigration massive, à l'accaparement des terres par la Compagnie de Londres, à l'ingérence du Parlement

33 La Minerve, 13 déc. 1832. Thomas Chapais, Cours d'histoire du Canada (Montréal, Ed. Bernard Valiquette, 1919) tome IV: 5, mentionne que «La Minerve se plaignait de ce que les magistrats eussent nommé des connétables spéciaux pour empêcher le désordre. Ces magistrats, en grande majorité, étaient d'une impartialité plus que douteuse. Plusieurs comptaient parmi les partisans de M. Bagg. Deux ou trois d'entre eux étaient membres du Conseil législatif qui avaient incarcéré $M$. Tracey durant la session».

34 The Canadian Courant, 19 mai 1832.

35 La Minerve, 24 mai 1832. 
impérial dans les affaires bas-canadiennes, thèmes abordés par Tracey tout au long de la campagne. Les suites de l'élection laissèrent voir comment, en 1832, les Canadiens n'étaient pas prêts à admettre que le véritable pouvoir se trouvait en Angleterre. Ils préféraient croire que c'était exclusivement les Britanniques installés à Montréal et ailleurs dans la province, plutôt que ceux résidant en Angleterre, qui cherchaient à s'accaparer tous les pouvoirs ou encore à conduire les destinées du pays. Les Canadiens s'indignèrent lorsque les gazettes anglaises leur prêtèrent l'intention de se révolter, de chercher à se défaire des liens qui les rattachaient à la mère patrie. Et même lorsque le roi convoqua pour un dîner le lieutenant-colonel McIntosh, officier qui avait donné l'ordre aux troupes de faire feu le $21 \mathrm{mai}$, et encore lorsqu'il le décora d'une croix d'honneur, les Canadiens voulurent croire que le roi avait été mal informé et mal conseillé. Pourtant, la façon dont les procédures judiciaires furent menées et la collusion des diverses autorités en vue d'innocenter les officiers et les magistrats, enfin, l'assentiment royal, tout cela prouvait bien que le pouvoir réel se trouvait à Londres.

Les problèmes ethniques, sociaux, économiques et politiques ne furent évidemment pas solutionnés par l'élection de 1832. Au contraire, celle-ci amena une aggravation de ces conflits, notamment à la suite de l'indignation ressentie par les Canadiens et par la confirmation, pour les Britanniques, du fait que leurs gestes seraient toujours approuvés par Londres. Les deux groupes s'enfonçaient donc toujours davantage dans leurs sentiments d'animosité et d'intolérance l'un vis-à-vis de l'autre, situation conflictuelle qui s'envenimera jusqu'aux événements de 1837.

L'élection partielle du Quartier-Ouest de Montréal, en 1832, et surtout les troubles du 21 mai, servirent en quelque sorte de cas exemplaire. Ils témoignèrent de l'acuité des oppositions et laissèrent présager la tournure des événements qui éclateraient cinq ans plus tard. Les agissements des magistrats et de la force militaire, les procédures judiciaires et la sanction de Londres, d'une part, les vains efforts des Canadiens pour obtenir la satisfaction de leurs griefs et leur statut de victimes, d'autre part, tout cela servit de répétition à 1837. 